\title{
Pythagorean triangles with sum of its two legs as Dodecic
}

\author{
Mita Darbari 1,* and Prashans Darbari ${ }^{2}$ \\ ${ }^{1}$ Department of Mathematics, St. Aloysius College (Autonomous), Jabalpur, Madhya Pradesh, India. \\ ${ }^{2}$ Department of Mathematics, BS-MS Programme, Indian Institute of Science Education \& Research, Mohali, Punjab, India.
}

GSC Advanced Engineering and Technology, 2022, 03(01), 011-015

Publication history: Received on 14 January 2022; revised on 15 February 2022; accepted on 15 February 2022

Article DOI: https://doi.org/10.30574/gscaet.2022.3.1.0028

\begin{abstract}
Number Theory is almost four thousand years old. In ancient clay tablet in Babylon, integral solutions of Pythagorean equations were listed down. Pythagoras theorem has always fascinated young and old mind alike. More than four hundred proofs of the Pythagoras theorem have been discovered so far. Pythagoras theorem is applied in almost every sphere of science- from geometry to Einstein's theory of relativity. New Pythagorean triangles are being discovered which satisfy certain constraints. Integral solutions of Diophantine equations related to Pythagorean equation are sought by many mathematicians. Applications of these triangles are also being explored in various fields of knowledge. In this paper, we have found nineteen extraordinary Pythagorean Triangles where their sum of two legs are dodecic numbers. These triangles are found by solving the dodecic Diophantine equation using the software Mathematica. Some interesting properties of these Pythagorean Triangles are also observed. Their applications can be explored in cryptography.
\end{abstract}

Keywords: Euclidean formula; Opposite Parity; Primitive Pythagorean Triangle; Dodecic

\section{Introduction}

The search for special Pythagorean Triangles continues till today. Darbari et al. [1] have discovered special Pythagorean Triangles with sum of their two legs as undecic. Darbari et al. [2] have found methods to use these Pythagorean Triangles in cryptography. Darbari and Darbari [3] have found out special Pythagorean Triangles in connection with Harshad numbers while Darbari and Darbari [4] have found exceptional Pythagorean Triangles with their perimeter as sum of three squares with two sides consecutive.

An attempt has been made to find special Pythagorean Triangles with sum of its two legs as a number which is a twelfth power of a number, i.e., is a dodecic.

\section{Definitions}

\subsection{Dodecic}

A number is called a dodecic if it is twelfth power of some number.

\subsubsection{Pythagorean Equation}

A quadratic equation

\footnotetext{
${ }^{*}$ Corresponding author: Mita Darbari

Department of Mathematics, St. Aloysius College (Autonomous), Jabalpur, Madhya Pradesh, India.

Copyright $@ 2022$ Author(s) retain the copyright of this article. This article is published under the terms of the Creative Commons Attribution Liscense 4.0
} 


$$
X^{2}+Y^{2}=Z^{2}
$$

is called Pythagorean equation [5] after the famous mathematician and philosopher Pythagoras. It still remains as one of the most important equations of the world in all times.

\subsubsection{Pythagorean Triangle [6]}

A right angled triangle with sides $X, Y$ and $Z$ is called Pythagorean Triangle if $X, Y$ and $Z$ are positive integers. $X$ and $Y$ are called its legs and $Z$ is called its hypotenuse. Pythagorean triangles satisfy Pythagorean equation $X^{2}+Y^{2}=Z^{2}$.

If $X, Y$ and $Z$ satisfy Pythagorean equation and $a$ is positive integer, then $a X, a Y$ and $a Z$ also satisfy it. Therefore, one Pythagorean Triangle can generate infinite Pythagorean triangles.

\subsubsection{Primitive Pythagorean Triangle}

A Pythagorean Triangle is said to be primitive if $X, Y$ and $Z$ are co-primes, i.e., their greatest common divisor is one. Or, in other words, $\operatorname{GCD}(X, Y, Z)=1$.

\subsection{Opposite Parity}

Two natural numbers $m$ and $n$ are called of opposite parity if one of them is even and other is odd, i.e., $m ¥ n$ ( $m o d 2$ ).

\subsubsection{Euclidean Formula [7]}

The positive primitive solutions of Pythagorean Equation with $Y$ even are

$$
X=m^{2}-n^{2}, Y=2 m n, Z=m^{2}+n^{2},
$$

where $m$ and $n$ are arbitrary integers of opposite parity with $m>n>0$ and $(m, n)=1$.

\section{Method of Analysis}

In the Pythagorean mathematics, primitive solutions of the Pythagorean Equation

$$
\mathrm{X}^{2}+\mathrm{Y}^{2}=\mathrm{Z}^{2}(1)
$$

is given by 2.6

$$
\mathrm{X}=m^{2}-n^{2}, \mathrm{Y}=2 m n, \mathrm{Z}=m^{2}+n^{2}(2)
$$

where $m, n \in$ I such that $m>n>0$ and $(m, n)=1$ with one of them is odd other even.

\subsection{Sum of two legs is a power of twelve, that is, dodecic}

If $\mathrm{X}$ and $\mathrm{Y}$ are two legs of a right angled triangle and $\mathrm{Z}$ is the hypotenuse, then

$$
\begin{gathered}
X+Y=\beta^{12}(3) \\
\Rightarrow m^{2}-n^{2}+2 m n=\beta^{12}(4)
\end{gathered}
$$

Solving this equation using the software Mathematica, the twelve solutions of (4) are given by

$$
\begin{aligned}
& \beta=\left(m^{2}-n^{2}+2 m n\right)^{1 / 12}, \beta=-\left(m^{2}-n^{2}+2 m n\right)^{1 / 12}, \beta=-\mathrm{i}\left(m^{2}-n^{2}+2 m n\right)^{1 / 12}, \\
& \beta=\mathrm{i}\left(m^{2}-n^{2}+2 m n\right)^{1 / 12}, \beta=-(-1)^{1 / 6}\left(m^{2}-n^{2}+2 m n\right)^{1 / 12}, \beta=(-1)^{1 / 6}\left(m^{2}-n^{2}+2 m n\right)^{1 / 12}, \\
& \beta=-(-1)^{1 / 3}\left(m^{2}-n^{2}+2 m n\right)^{1 / 12}, \beta=(-1)^{1 / 3}\left(m^{2}-n^{2}+2 m n\right)^{1 / 12}, \beta=-(-1)^{2 / 3}\left(m^{2}-n^{2}+2 m n\right)^{1 / 12}, \\
& \beta=(-1)^{2 / 3}\left(m^{2}-n^{2}+2 m n\right)^{1 / 12}, \beta=-(-1)^{5 / 6}\left(m^{2}-n^{2}+2 m n\right)^{1 / 12}, \beta=(-1)^{5 / 6}\left(m^{2}-n^{2}+2 m n\right)^{1 / 12} .
\end{aligned}
$$

Solving the integral solutions, using Mathematica, using the command 
Find Instance $\left[m^{2}-n^{2}+2 m n-\beta^{12}==0 \& \& \mathrm{n}<\mathrm{m} \& \& 0<\mathrm{m}<10^{15} \& \& 0<\mathrm{n}<10^{15} \& \& 0<\beta<10^{11} \& \& \mathrm{GCD}[\mathrm{m}, \mathrm{n}]==1, \quad\{\mathrm{~m}, \mathrm{n}, \beta\}\right.$, Integers,10000], we get only 19 solutions. They are as follows:

Table 1 Values of $m, n, \beta$

\begin{tabular}{|l|l|l|l|}
\hline S. N. & $\mathbf{m}$ & $\mathbf{n}$ & $\mathbf{B}$ \\
\hline 1 & 83221 & 80030 & 7 \\
\hline 2 & 17555585 & 11743968 & 17 \\
\hline 3 & 144336505 & 3796728 & 23 \\
\hline 4 & 707744245 & 244990478 & 31 \\
\hline 5 & 4307805349 & 493243310 & 41 \\
\hline 6 & 7754194705 & 5738360402 & 47 \\
\hline 7 & 13330535741 & 531116422 & 49 \\
\hline 8 & 104082680521 & 31579397642 & 71 \\
\hline 9 & 120865698101 & 41398812492 & 73 \\
\hline 10 & 221522493785 & 23904378538 & 79 \\
\hline 11 & 428902751369 & 81160408968 & 89 \\
\hline 12 & 622352222549 & 338094915118 & 97 \\
\hline 13 & 940927902041 & 353620702330 & 103 \\
\hline 14 & 1669585699885 & 555803980548 & 113 \\
\hline 15 & 2400863098325 & 539668450092 & 119 \\
\hline 16 & 2475813102101 & 427628706622 & 119 \\
\hline 17 & 3016237805729 & 2248102736098 & 127 \\
\hline 18 & 5469525155081 & 1455204818410 & 137 \\
\hline 19 & 8876744496929 & 4744194947088 & 151 \\
\hline
\end{tabular}

\subsection{Perimeter is a sum of two squares and a dodecic}

Table 2 Values of X, Y, Z

\begin{tabular}{|l|l|l|l|}
\hline $\mathbf{S . N}$. & $\mathbf{X}=\mathbf{m}^{\mathbf{2}}-\mathbf{n}^{\mathbf{2}}$ & $\mathbf{Y}=\mathbf{2} \mathbf{m n}$ & $\mathbf{Z}=\mathbf{m}^{\mathbf{2}+\mathbf{n}^{\mathbf{2}}}$ \\
\hline 1 & 520933941 & 13320353260 & 13330535741 \\
\hline 2 & 170277780307201 & 412344456922560 & 446119349077249 \\
\hline 3 & 20818611532109041 & 1096012899911280 & 20847441819121009 \\
\hline 4 & 440881582019951541 & 346781201768598220 & 560922250641288509 \\
\hline 5 & 18313897962013255701 & 4249592338352930380 & 18800475887732767901 \\
\hline 6 & 27198755419808435421 & 88992727689140142820 & 93056315626291638629 \\
\hline 7 & 177421098488360336997 & 14160132892206077404 & 177985267795796501165 \\
\hline 8 & 9835946029004997671277 & 6573736711635813462964 & 11830462739868107991605 \\
\hline 9 & 12894655301694299756137 & 10007392744795958955384 & 16322378653189850256265 \\
\hline 10 & 48500795939437368608781 & 10590715092236784772660 & 49643634566013358643669 \\
\hline 11 & 177370558148045216549137 & 69619845417216923754384 & 190544582115751246199185 \\
\hline 12 & 273014117283032399103477 & 420828243712405601191564 & 501630460540327649011325 \\
\hline 13 & 760297715722915224536781 & 665463171123263720911060 & 1010392917955640159394581 \\
\hline 14 & 2478598344467483726632921 & 1855924755724203011673960 & 3096434474053486851393529 \\
\hline
\end{tabular}




\begin{tabular}{|l|l|l|l|}
\hline 15 & 5472901580874017122997161 & 2591340134312259502591800 & 6055385652923420112614089 \\
\hline 16 & 5946784205807772103963317 & 2117457509378504521625644 & 6312516827262181197265085 \\
\hline 17 & 4043724588657578912955837 & 13561624927563185359010884 & 14151656412760206577487045 \\
\hline 18 & 27798084358540156025188461 & 15918558720177147387682420 & 32033326485587518175044661 \\
\hline 19 & 56289207167783974170751297 & 84226012777843544666985504 & 101303978559734596410110785 \\
\hline
\end{tabular}

From equation (2), hypotenuse is $\mathrm{Z}=m^{2}+n^{2}$ and from equation (3),

$X+Y=\beta^{12}$

Therefore, perimeter is given by

$\mathrm{X}+\mathrm{Y}+\mathrm{Z}=\beta^{12}+m^{2}+n^{2}$

Substituting the values of $\mathrm{m}, \mathrm{n}$ from Table 1, we find the values of $\mathrm{X}, \mathrm{Y}$ and $\mathrm{Z}$ from equation $(2) ; \mathrm{X}=m^{2}-n^{2}, \mathrm{Y}=2 m n$,

$\mathrm{Z}=m^{2}+n^{2}$

The following table verifies that the perimeter is a sum of two squares and a dodecic,

Table $3 X+Y+Z=\beta^{12}+m^{2}+n^{2}$

\begin{tabular}{|l|l|}
\hline S.N. & $\mathbf{X}+\mathbf{Y}+\mathbf{Z}=\boldsymbol{\beta}^{12}+\mathbf{m}^{2}+\mathbf{n}^{\mathbf{2}}$ \\
\hline 1 & $27171822942=7^{12}+83221^{2}+80030^{2}$ \\
\hline 2 & $1028741586307010=17^{12}+17555585^{2}+11743968^{2}$ \\
\hline 3 & $42762066251141330=23^{12}+144336505^{2}+3796728^{2}$ \\
\hline 4 & $1348585034429838270=31^{12}+707744245^{2}+244990478^{2}$ \\
\hline 5 & $41363966188098953982=41^{12}+4307805349^{2}+493243310^{2}$ \\
\hline 6 & $209247798735240216870=47^{12}+7754194705^{2}+5738360402^{2}$ \\
\hline 7 & $369566499176362915566=4912+13330535741^{2}+531116422^{2}$ \\
\hline 8 & $28240145480508919125846=71^{12}+104082680521^{2}+31579397642^{2}$ \\
\hline 9 & $39224426699680108967786=73^{12}+120865698101^{2}+41398812492^{2}$ \\
\hline 10 & $108735145597687512025110=7912+221522493785^{2}+239043785382$ \\
\hline 11 & $437534985681013386502706=89^{12}+428902751369^{2}+81160408968^{2}$ \\
\hline 12 & $1195472821535765649306366=97^{12}+622352222549^{2}+338094915118^{2}$ \\
\hline 13 & $2436153804801819104842422=103^{12}+940927902041^{2}+353620702330^{2}$ \\
\hline 14 & $7430957574245173589700410=113^{12}+1669585699885^{2}+555803980548^{2}$ \\
\hline 15 & $14119627368109696738203050=119^{12}+2400863098325^{2}+539668450092^{2}$ \\
\hline 16 & $14376758542448457822854046=119^{12}+2475813102101^{2}+427628706622^{2}$ \\
\hline 17 & $31757005928980970849453766=12712+3016237805729^{2}+2248102736098^{2}$ \\
\hline 18 & $75749969564304821587915542=137^{12}+5469525155081^{2}+1455204818410^{2}$ \\
\hline 19 & $241819198505362115247847586=151^{12}+8876744496929^{2}+4744194947088^{2}$ \\
\hline
\end{tabular}




\section{Results and discussion}

We observe that

- $\quad$ The value 119 of $\beta$ is repeated once.

- $\quad$ Except for 49 and repeated value 119, which are multiple of 7, rest other values of $\beta$ are prime numbers.

- $\quad 2 m^{2}+m n=0(\bmod 2)$

- $4 m^{2}+2 m n=0(\bmod 4)$

- $\quad 3 m^{2}+m n+n^{2}=0(\bmod 3)$

- $m+n+\beta=0(\bmod 4)$ or $2(\bmod 4)$

- $\quad 3 m+5 n+\beta=0(\bmod 2)$

- $6 m^{3}+3 n^{3}+\Phi(\beta)=0(\bmod 6)$ or $4(\bmod 6)$

- $\mathrm{m}^{2}+\mathrm{n}^{2}+\{\Phi(\beta)\}^{2}=1(\bmod 4)$

- $\mathrm{m}^{3}+\mathrm{n}^{3}+\{\Phi(\beta)\}^{3}=1(\bmod 4)$

- $\mathrm{m}^{4}+\mathrm{n}^{4}+\{\Phi(\beta)\}^{4}=1(\bmod 4)$

- $\quad \mathrm{X}+\mathrm{Y}+\mathrm{Z}=0(\bmod 2)$

- $\quad 2 X+Y+Z=0(\bmod 3)$

- $\quad X+2 Y+3 Z=0(\bmod 4)$

- $\quad(\mathrm{Y}+\mathrm{Z}-\mathrm{X})^{2}=2(\mathrm{Y}+\mathrm{Z})(\mathrm{Z}-\mathrm{X})$

- $\quad(\mathrm{X}+2 \mathrm{Y}+\mathrm{Z})^{2}=(\mathrm{Z}-\mathrm{X})^{2}+4(\mathrm{X}+\mathrm{Y})(\mathrm{Y}+\mathrm{Z})$

- $\quad \mathrm{X}+2 \mathrm{Y}+\mathrm{Z} \pm 2\{(\mathrm{X}+\mathrm{Y})(\mathrm{Z}-\mathrm{Y})\}^{1 / 2}=0(\bmod 16)$ or $=0(\bmod 4)$

\section{Conclusion}

In conclusion, one may endeavor to discover Pythagorean Triangle which satisfy the conditions similar to that presented in the above problem.

\section{Compliance with ethical standards}

Disclosure of conflict of interest

No conflict of interest to disclosed.

\section{References}

[1] Darbari M and Darbari P. (2019). Special pythagorean triangles with sum of their two legs as undecic. International Journal of Innovative Technology and Exploring Engineering, 8(11), 2019-2023.

[2] Darbari M, Darbari P, Nema S, Sahu M and Soni R. (2019). Fortifying the messages. International Journal of Scientific \& Technology Research, 8(12), 544-552.

[3] Darbari M, Darbari P. (2020). Harshad numbers and pythagorean triangles. In: Arasu V and Darbari M (Eds), Deliberations on Advances in Physical, Mathematical and Computational Sciences. Shree Vinayak Publication, Agra, 137-143.

[4] Darbari M and Darbari P. (2019). Some Special Pythagorean Triangles. Journal of Emerging Technologies and Innovative Research, 6(2), 135-139.

[5] Robbins N. (2006). Beginning Number Theory, Second edition. Jones and Bartlett, Sudbury. Canada, 2.

[6] Niven I, Zuckerman HS and Montgomery HL. (2018). An Introduction to the Theory of Numbers, Fifth edition. Wiley India, New Delhi, 231.

[7] Posamentier AS. (2010). The Pythagorean Theorem: The Story of its Power and Beauty. Prometheus Books, First edition, New York, 127-128. 